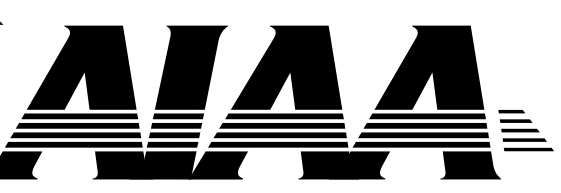

AIAA-2008-2085

Sensitivity Enhancement of Modal

Frequencies for Sensing using

System Augmentation and Optimal

Feedback Auxiliary Signals

Kiran D'Souza and Bogdan I. Epureanu

Department of Mechanical Engineering

University of Michigan, Ann Arbor, MI 48109

control input

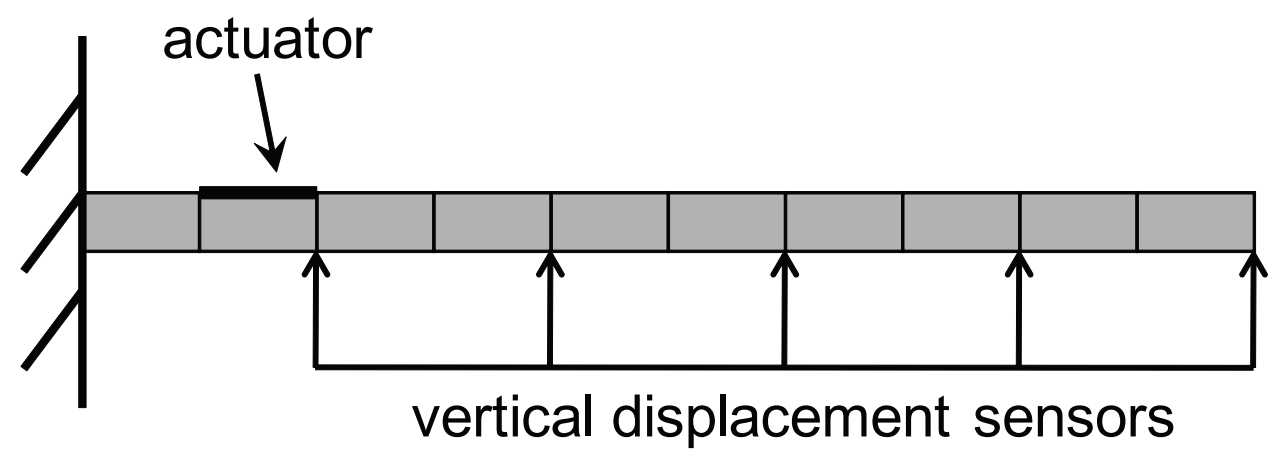

49th AIAA/ASME/ASCE/AHS/ASC Structures, Structural Dynamics and Materials

Conference, Chicago, Illinois, April 2008 


\title{
Sensitivity Enhancement of Modal Frequencies for Sensing using System Augmentation and Optimal Feedback Auxiliary Signals
}

\author{
Kiran D'Souza and Bogdan I. Epureanu* \\ Department of Mechanical Engineering \\ University of Michigan, Ann Arbor, MI 48109
}

\begin{abstract}
Changes in resonant frequencies of linear systems due to added mass and loss of stiffness have been explored extensively for applications such as sensing, system identification and damage detection. The benefits of using resonant frequencies are the small number of sensors required and their insensitivity to noise (as opposed to using other system properties such as mode shapes or mode shape curvatures). However, resonant frequencies are also insensitive to damages/perturbations in the system. To overcome this limitation, linear feedback excitations have been used to place the poles (and eigenvectors) of the system and to increase their sensitivity to changes in the system properties. Recently, system augmentation has been combined with optimal nonlinear feedback auxiliary signals (as applied to damage detection). That method extended the use of sensitivity enhancement of resonant frequencies to nonlinear systems. Linear systems were also considered by using nonlinear feedback to demonstrate the benefits of nonlinear feedback auxiliary signals with respect to traditional linear feedback excitation. Nonlinear feedback auxiliary signals have been designed to maximize the sensitivity of resonant frequencies to changes in stiffness (while minimizing the control effort). The nonlinearities of the closed loop system were handled by forming augmented linear systems. In this work, this approach is refined to improve its robustness. In particular, an optimization is employed not only to maximize the sensitivity of resonant frequencies to added mass at particular locations, but also to detect changes in temperature and humidity. Additionally, a methodology is presented to accurately extract augmented frequencies from displacement and forcing data corrupted by noise. Numerical simulations of a nonlinear cantilevered beam are performed and the effects of measurement noise are discussed.
\end{abstract}

\begin{tabular}{|c|c|}
\hline & Nomenclature \\
\hline B & control input matrix \\
\hline $\mathbf{K}_{C}$ & full gain matrix \\
\hline $\mathbf{K}_{C L}, \mathbf{K}_{C N}$ & $\begin{array}{l}\text { linear and nonlinear portions of the } \\
\text { gain matrix }\end{array}$ \\
\hline $\mathbf{K}_{C L A}, \mathbf{K}_{C N A}$ & $\begin{array}{l}\text { augmented linear and nonlinear } \\
\text { portions of the gain matrix }\end{array}$ \\
\hline $\mathbf{M}, \mathbf{D}, \mathbf{K}$ & $\begin{array}{l}\text { linear mass, damping and stiffness } \\
\text { matrices }\end{array}$ \\
\hline $\mathbf{N}_{I}, \mathbf{N}_{D}, \mathbf{N}_{S}$ & $\begin{array}{l}\text { nonlinear inertia, damping and } \\
\text { stiffness (parameter) matrices }\end{array}$ \\
\hline $\mathbf{N}_{C I}, \mathbf{N}_{C D}, \mathbf{N}_{C S}$ & $\begin{array}{l}\text { coupled inertia, damping and } \\
\text { stiffness (parameter) matrices }\end{array}$ \\
\hline $\mathbf{N}_{A I}, \mathbf{N}_{A D}, \mathbf{N}_{A S}$ & $\begin{array}{l}\text { augmented inertia, damping and } \\
\text { stiffness (parameter) matrices }\end{array}$ \\
\hline $\mathbf{S}$ & first order sensitivity matrix \\
\hline g & external excitation \\
\hline h & augmented forcing \\
\hline $\mathbf{p}$ & vector of parameters of the system \\
\hline$q$ & number of measured frequencies \\
\hline$r$ & number of varying parameters \\
\hline$t$ & number of controller configurations \\
\hline
\end{tabular}

${ }^{*}$ Copyright (C) 2008 by K. D'Souza and B. I. Epureanu. Published by the American Institute of Aeronautics and Astronautics, Inc. with permission. Corresponding author: B. I. Epureanu, epureanu@umich.edu

\author{
x coordinate vector \\ $\mathbf{y} \quad$ vector of augmented variables \\ $\omega \quad$ resonant frequency of the system
}

\section{Introduction}

Vibration-based identification of changes in structural parameters is currently used in a wide variety of technologies. In particular, two areas, sensing and damage detection, focus closely on identifying parameter variations such as mass and stiffness by exploiting variations in resonant frequencies. For example, recent sensing techniques for chemical and biological detection as well as atomic force microscopes in tapping mode ${ }^{1}$ use the vibration of micro-structures such as micro-beams ${ }^{2}$ and micro-cantilevers. ${ }^{3-5}$

Resonant frequencies are used not only for micro-scale systems but also for monitoring large-scale structures such as bridges, space and aircraft. Similar to sensing, vibration-based damage detection ${ }^{6-8}$ uses changes in the systems modal properties to identify parameter variations indicative of damage. Some of these damage detection techniques use both mode shapes and natural frequencies, although measuring mode shapes is more sensitive to noise ${ }^{9}$ than measuring frequencies, and requires more measurements.

Sensing and detection methods that use only the frequencies of the system (which herein are referred to as 


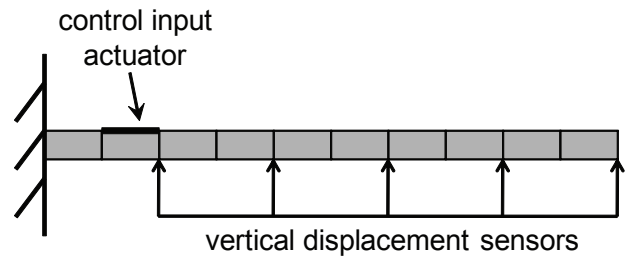

Fig. 1 Linear beam excited by one piezoelectric patch using nonlinear feedback auxiliary signals from 5 sensors.

frequency-shift based methods ${ }^{10}$ ) have recently become of increasing interest. They have been developed because frequency extraction can be done robustly for both micro- and large-scale applications.

There are two central drawbacks to frequency-shift based methods. The first drawback is that only a limited number of frequencies can be measured accurately, which leads to an under-determined problem when solving for multiple different parameter variations (e.g. damage scenarios, ${ }^{11,12}$ or sensor outputs). To overcome this problem, in the context of damage detection, Nalitolela et al. ${ }^{13}$ proposed to extract additional modal frequencies by adding mass or stiffness to the structure. However, in practice, the physical addition of mass or stiffness is difficult to implement. This difficulty was overcome by Lew and Juang ${ }^{14}$ by introducing virtual passive controllers. They used controllers to generate additional vibration frequencies in the closed loop system instead of attaching physical mass or stiffness elements to the structure.

The second drawback of frequency-shift based methods is that the sensitivity of the lowest frequencies to parameter variations is often quite low. Therefore, in sensing applications, the sensitivity of the sensors can be too low; and in damage detection applications, the lowest damage that can be identified is exceedingly large. For example, Swamidas and Chen ${ }^{15}$ showed this in a finite element study of a cracked plate. In their study, a surface crack $40 \%$ the width of the plate and $70 \%$ through its depth had a maximum frequency shift of less than $0.7 \%$. Adams et al. ${ }^{16}$ demonstrated this low sensitivity experimentally using an aluminum bar under axial loading. They found less than a $1 \%$ change in the first three frequencies when they used a saw to make a cut through $30 \%$ of the beam's surface area near the beam's center.

To overcome the insensitivity of the frequencies to parameter variations, Ray and Tian ${ }^{17}$ proposed sensitivity enhancing feedback control. They applied closed loop vibration control in smart structures for pole placement with the objective of increasing the sensitivity of resonant frequencies to changes in the system. That method was demonstrated through numerical simulations of a cantilevered beam. Experimental validation of sensitivity enhancing feedback control was conducted by Ray et al. ${ }^{18}$ on a cantilevered beam in bending. Ray and Marini ${ }^{19}$ developed an optimization method to minimize the control effort while maximizing frequency sensitivity for a single fixed actuator location. Juang et al. ${ }^{20}$

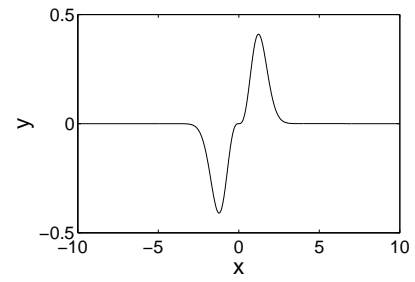

Fig. 2 Nonlinearity used in the nonlinear controllers.

proposed an eigenstructure assignment technique that is useful in extending sensitivity enhancing control from single input to multi-input systems. Since in the multiinput case there are an infinite number of placement options for the modal frequencies, they choose the output feedback with the lowest control effort. They achieve this by using the open loop eigenvectors as the desired values of the closed loop eigenvectors, which leads to minimum control gains and minimum control effort. To address the limited frequency information drawback, Koh and Ray $^{21}$ proposed the use of multiple independent closed loop systems. Jiang et al. ${ }^{22}$ developed an optimizing algorithm for placement of the frequencies and eigenvectors to maximize frequency sensitivity and minimize the control effort in the multi-input case.

One of the frontiers for the development of sensors and the advancement of damage detection technologies is tackling nonlinear systems. In these technologies, nonlinearities are often unavoidable during the regular vibration of the system, and hence, they have to be accounted for. Furthermore, they can be exploited for enhancing sensitivity. For example, recently sensitivity enhancing control has been proposed for nonlinear systems. ${ }^{23,24}$ The nonlinear systems were handled by forming higher dimensional augmented linear systems, ${ }^{23-27}$ which are designed to follow a single trajectory of the nonlinear system. The idea of optimal augmentations has also been introduced by the authors. ${ }^{23}$ The types of nonlinearities explored have included cubic spring nonlinearities ${ }^{23-27}$ and Coulomb friction. ${ }^{26}$

In this work, a linear beam is explored using optimal system augmentations and nonlinear feedback auxiliary signals. The objective here is to build on the work of the authors ${ }^{23,24}$ for the case where there are only limited measurements available and a single input actuator. In this work, the motion of the structure, which must be fed back into the system using the control gain matrix, is known only at 5 locations. Linear approaches would allow for the placement of only 2 resonant frequencies, which would not be sufficient for many practical purposes. In contrast, the use of nonlinear feedback auxiliary signals allows the creation of several augmented variables, which increases the amount of measurement information, and in turn enables the placement of additional frequencies of the system. Various numerical simulations are included to demonstrate the proposed techniques, and to discuss the effects of random noise. 


\begin{tabular}{|c|c|c|c|}
\hline $\begin{array}{c}\text { Exact Augmented } \\
\text { Eigenvalues }\end{array}$ & $\begin{array}{c}\text { Augmented Eigenvalues } \\
\text { Extracted by DSPI (no noise) }\end{array}$ & $\begin{array}{c}\text { Average Augmented } \\
\text { Eigenvalues Extracted by DSPI } \\
\text { (noisy measurements) }\end{array}$ & $\begin{array}{c}\text { Standard Deviation of } \\
\text { Augmented Frequencies } \\
\text { Extracted by DSPI }\end{array}$ \\
\hline$-50.449+320.8487 \mathrm{i}$ & $-50.4304+320.845 \mathrm{i}$ & $-56.0636+320.6479 \mathrm{i}$ & 2.4056 \\
$-51.1395+1887.875 \mathrm{i}$ & $-51.2038+1887.8741 \mathrm{i}$ & $-51.7111+1887.4735 \mathrm{i}$ & 0.3035 \\
$-59.0027+2031.5986 \mathrm{i}$ & $-58.9349+2031.5773 \mathrm{i}$ & $-60.7222+2033.4844 \mathrm{i}$ & 0.625 \\
$-135.8709+3658.8146 \mathrm{i}$ & $-135.7516+3658.7827 \mathrm{i}$ & $-135.8077+3659.1526 \mathrm{i}$ & 0.4331 \\
$-260.9331+5581.6613 \mathrm{i}$ & $-261.0341+5581.6112 \mathrm{i}$ & $-260.8428+5581.6889 \mathrm{i}$ & 0.558 \\
$-510.741+9425.9538 \mathrm{i}$ & $-510.6796+9426.1458 \mathrm{i}$ & $-511.3594+9426.7778 \mathrm{i}$ & 1.1731 \\
$-959.1626+13960.3522 \mathrm{i}$ & $-958.6539+13960.5427 \mathrm{i}$ & $-959.5121+13960.5772 \mathrm{i}$ & 3.216 \\
\hline
\end{tabular}

Table 1 First 7 eigenvalues of a baseline (nominal) closed loop system.

\section{Methodology}

In this section, the procedure for sensitivity enhancement using nonlinear feedback auxiliary signals in linear systems is presented. First, an overview of system augmentation with feedback auxiliary signals is provided. Next, the frequency-shift based detection procedure is outlined. Finally, the augmented frequency extraction procedure is detailed.

\section{System Augmentation}

In this section, a brief overview of system augmentation is provided. First, an example of a one degree of freedom system containing 2 nonlinearities is discussed. Then, the general form of the augmented equations are presented for a controlled system. Finally, an example of a simple controlled system is included. More details on system augmentation can be found in previous work by the authors. ${ }^{23-27}$

Consider a mass connected to the ground by a linear, cubic and quintic spring. The equation of motion for this nonlinear system is given by

$$
m \ddot{x}+k x+k_{n 1} x^{3}+k_{n 2} x^{5}=g(t),
$$

where $x$ is the position of the mass $m, g(t)$ is the external excitation and $k, k_{n 1}$, and $k_{n 2}$ are the linear, cubic and quintic spring stiffnesses, respectively.

The fundamental idea behind the augmentation is that higher dimensional augmented linear systems can be designed to follow a single trajectory of a nonlinear system. For the nonlinear system in Eq. 1, a higher dimensional augmented linear system can be formed by adding an additional degree of freedom for each nonlinearity to obtain the augmented equations of motion as

$$
\begin{aligned}
m \ddot{x}+k x+k_{n 1} y_{1}+k_{n 2} y_{2} & =g(t), \\
m_{a 1} \ddot{y}_{1}+k_{c 1} x+k_{a 1} y_{1} & =h_{1}(t), \\
m_{a 2} \ddot{y}_{2}+k_{c 2} x+k_{a 2} y_{2} & =h_{2}(t),
\end{aligned}
$$

where $y_{1}=x^{3}$ and $y_{2}=x^{5}$, with $m_{a i}, k_{a i}, k_{c i}, h_{i}(t)$, and $y_{i}$ corresponding to the augmented mass, augmented stiffness, coupled stiffness, augmented forcing, and augmented variable, respectively.

Typically, the parameters $k_{c i}$ are chosen to maintain the symmetry of the system $\left(k_{c 1}=k_{n 1}\right.$ and $\left.k_{c 2}=k_{n 2}\right)$, $m_{a i}$ are chosen similar to the mass at the degree of freedom they are coupled to $\left(m_{a 1}=m_{a 2}=m\right)$, and $k_{a i}$ are chosen to be low multiples of the nonlinear spring stiffness $\left(k_{a 1}=\xi_{1} k_{n 1}\right.$ and $\left.k_{a 2}=\xi_{2} k_{n 2}\right)$. However, one can choose these parameters to optimally suit their needs. An optimization of the augmentation for sensitivity enhancement has been established. ${ }^{23,24}$ It uses the typical values of the augmentation as the starting point and then optimizes the parameters $k_{c i}$ and $k_{a i}$ by finding the optimal control gains in the augmented equations. Additionally, a separate parameter is included in the optimization to adjust the augmented mass.

The augmented variables $y_{i}$ can be computed directly from $x\left(y_{1}=x^{3}\right.$ and $\left.y_{2}=x^{5}\right)$, and the augmented forcing $h_{i}(t)$ can be computed directly from the left hand side in Eq. 2. The specific form of the augmented forcing is a key feature in the augmentation because it ensures that, if the trajectory of the augmented linear system is projected onto the original (physical) space, it will follow the trajectory of the nonlinear system. Due to the required augmented forcing, the modal extraction technique used must be an input/output technique (as opposed to an output only approach).

There are several features of an augmented system that differ from a typical linear system and have to be considered when designing a controller for system interrogation. Consider the general equations of motion of an augmented system with a controller expressed as

$$
\begin{gathered}
{\left[\begin{array}{ll}
\mathbf{M} & \mathbf{N}_{I} \\
\mathbf{N}_{C I} & \mathbf{N}_{A I}
\end{array}\right]\left[\begin{array}{l}
\ddot{\mathbf{x}} \\
\ddot{\mathbf{y}}
\end{array}\right]+} \\
{\left[\begin{array}{ll}
\mathbf{D} & \mathbf{N}_{D} \\
\mathbf{N}_{C D} & \mathbf{N}_{A D}
\end{array}\right]\left[\begin{array}{l}
\dot{\mathbf{x}} \\
\dot{\mathbf{y}}
\end{array}\right]+} \\
{\left[\begin{array}{ll}
\mathbf{K} & \mathbf{N}_{S} \\
\mathbf{N}_{C S} & \mathbf{N}_{A S}
\end{array}\right]\left[\begin{array}{l}
\mathbf{x} \\
\mathbf{y}
\end{array}\right]+} \\
\mathbf{B}\left[\begin{array}{ll}
\mathbf{K}_{C L} & \mathbf{K}_{C N} \\
\mathbf{K}_{C L A} & \mathbf{K}_{C N A}
\end{array}\right]\left[\begin{array}{l}
\mathbf{x} \\
\mathbf{y}
\end{array}\right]
\end{gathered}
$$

where $\mathbf{M}, \mathbf{D}$ and $\mathbf{K}$ are the linear mass, damping and stiffness matrices; $\mathbf{N}_{I}, \mathbf{N}_{D}$, and $\mathbf{N}_{S}$ are nonlinear parameter matrices which contain terms such as the cubic and quintic stiffness terms; $\mathbf{N}_{C I}, \mathbf{N}_{C D}$, and $\mathbf{N}_{C S}$ are the coupled inertia, damping and stiffness matrices, which traditionally have been used to maintain the symmetry 

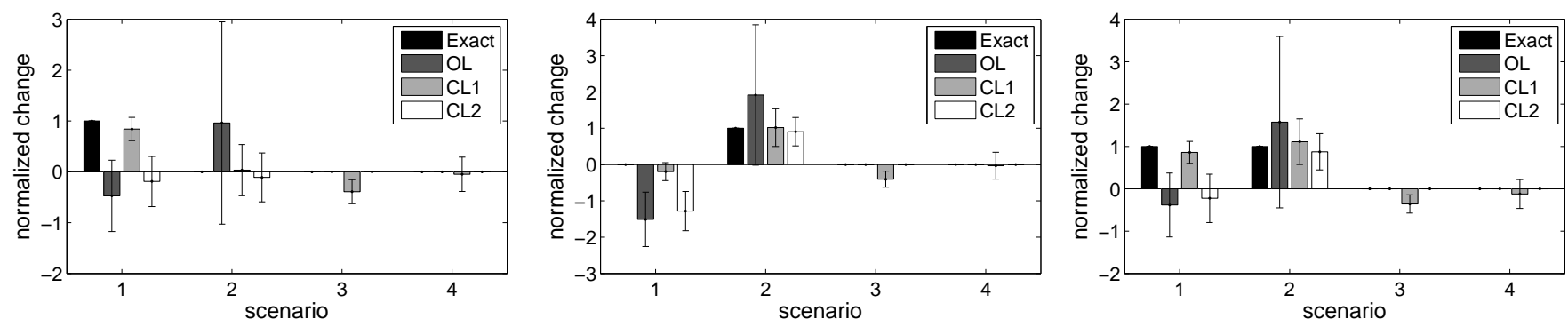

Fig. 3 Sensed mass by the open loop system $(O L)$, a closed loop system designed to detect humidity and temperature ( $C L 1)$, and a closed loop system not designed to detect humidity and temperature (CL2) for a system with mass added at the tip (left), midspan (center) and both locations (right). Scenario 1 represents changes in mass at the tip. Scenario 2 represents changes in mass at the midspan. Scenario 3 represents changes in humidity. Scenario 4 represents changes in temperature.

of the system; and $\mathbf{N}_{A I}, \mathbf{N}_{A D}$, and $\mathbf{N}_{A S}$ are the augmented parameter matrices which contain terms such as $m_{a i}$ and $k_{a i}$. B is the control input matrix, which has nonzero values in rows where there are input actuators that can excite the system. An interesting advantage of augmented systems is that all the rows of the augmentation can have nonzero entries in $\mathbf{B}$ because they do not require any physical actuation. The gain matrix $\mathbf{K}_{C}$ has been split into four parts in Eq. 3. The purely linear portion of the controller is given by $\mathbf{K}_{C L}$. The nonlinear portion of the controller is given by $\mathbf{K}_{C N}$. If a linear controller is desired $\mathbf{K}_{C N}$ must be set to zero. Finally, $\mathbf{K}_{C L A}$ and $\mathbf{K}_{C N A}$ are the augmented portions of the gain matrix. Again, no actual physical actuation is required in the augmented portion of the controller. Rather, the calculated actuation is used in the computation of the augmented forcing $\mathbf{h}$.

Next, consider the augmented system discussed in Eq. 2 controlled by a single point actuator

$$
\begin{aligned}
m \ddot{x}+k x+k_{n 1} y_{1}+k_{n 2} y_{2}+ & \\
K_{C L} x+K_{C N 1} y_{1}+K_{C N 2} y_{2} & =g(t), \\
m_{a 1} \ddot{y}_{1}+k_{c 1} x+k_{a 1} y_{1}+ & \\
K_{C L A 1} x+K_{C N A 1} y_{1} & =h_{1}(t), \\
m_{a 2} \ddot{y}_{2}+k_{c 2} x+k_{a 2} y_{2}+ & \\
K_{C L A 2} x+K_{C N A 2} y_{2} & =h_{2}(t) .
\end{aligned}
$$

The nonlinear actuation applied to the physical system is given by $K_{C N 1} y_{1}$ and $K_{C N 2} y_{2}$. Since augmented parameters (such as $k_{c i}$ and $k_{a i}$ ) are chosen by the user, they can incorporate the augmented controller gains to obtain the following augmented equations of motion

$$
\begin{aligned}
& m_{a 1} \ddot{y}_{1}+k_{c 1}^{\prime} x+k_{a 1}^{\prime} y_{1}=h_{1}(t), \\
& m_{a 2} \ddot{y}_{2}+k_{c 2}^{\prime} x+k_{a 2}^{\prime} y_{2}=h_{2}(t),
\end{aligned}
$$

where $k_{c 1}^{\prime}=k_{c 1}+K_{C L A 1}, k_{a 1}^{\prime}=k_{a 1}+K_{C N A 1}, k_{c 2}^{\prime}=$ $k_{c 2}+K_{C L A 2}$, and $k_{a 2}^{\prime}=k_{a 2}+K_{C N A 2}$.

The procedure for calculating the gain matrix $\mathbf{K}_{C}$ (containing linear and nonlinear control gains) consists of following an optimization algorithm previously established for augmented systems ${ }^{23,24}$ and linear systems. ${ }^{22}$
The procedure uses an eigenstructure assignment technique to place the eigenvectors and resonant frequencies of the augmented system. The optimization algorithm was developed to maximize the sensitivity of resonant frequencies to specified parameters in the system, while minimizing the control (actuation) effort given a set of constraints. The first constraint is that the linearized physical system must be stable (the fictitious augmented system can be unstable, however). The linearized system must be stable for the baseline (nominal) case and after the maximum changes in each of the specified parameters. The second constraint is that the change in resonant frequencies due to changes in the parameters of interest maintain a linear relationship over the designated parameter ranges. This constraint is enforced so that the approximations made in the first order frequency-shift based detection method are followed, as discussed in the next section.

\section{Frequency-Shift Based Detection Method}

In this section, the frequency-shift based detection method used in this work is outlined. The method is a first order perturbation method and has been used previously with sensitivity enhancing control. ${ }^{21-23}$ Essentially, the idea is to relate the changes in the modal frequencies $\delta \omega$ to the changes in certain parameters $\delta \mathbf{p}$ (e.g. stiffness, mass, damping parameters). Generally, the relationship between $\delta \mathbf{p}$ and $\delta \omega$ is nonlinear. However, it can be linearized to a first order perturbation form as

$$
\delta \omega=\mathbf{S} \delta \mathbf{p}
$$

where

$$
\begin{aligned}
\mathbf{S} & =\left[\begin{array}{cccc}
\frac{\partial \omega_{1}}{\partial p_{1}} & \frac{\partial \omega_{1}}{\partial p_{2}} & \ldots & \frac{\partial \omega_{1}}{\partial p_{r}} \\
\frac{\partial \omega_{2}}{\partial p_{1}} & \frac{\partial \omega_{2}}{\partial p_{2}} & \ldots & \frac{\partial \omega_{2}}{\partial p_{r}} \\
\vdots & \vdots & \ddots & \vdots \\
\frac{\partial \omega_{q}}{\partial p_{1}} & \frac{\partial \omega_{q}}{\partial p_{2}} & \ldots & \frac{\partial \omega_{q}}{\partial p_{r}}
\end{array}\right] \\
\approx & {\left[\begin{array}{cccc}
\frac{\Delta \omega_{1}}{\Delta p_{1}} & \frac{\Delta \omega_{1}}{\Delta p_{2}} & \ldots & \frac{\Delta \omega_{1}}{\Delta p_{r}} \\
\frac{\Delta \omega_{2}}{\Delta p_{1}} & \frac{\Delta \omega_{2}}{\Delta p_{2}} & \ldots & \frac{\Delta \omega_{2}}{\Delta p_{r}} \\
\vdots & \vdots & \ddots & \vdots \\
\frac{\Delta \omega_{q}}{\Delta p_{1}} & \frac{\Delta \omega_{q}}{\Delta p_{2}} & \cdots & \frac{\Delta \omega_{q}}{\Delta p_{r}}
\end{array}\right], }
\end{aligned}
$$



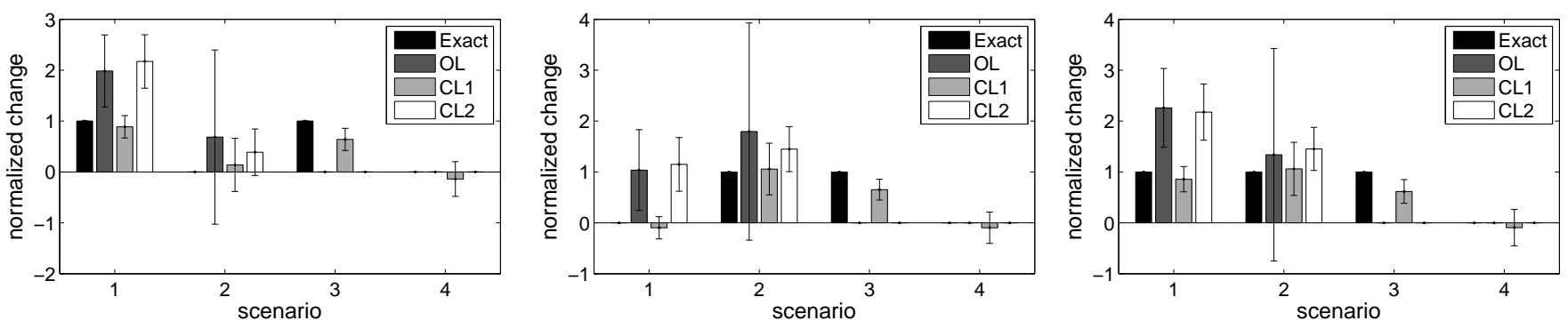

Fig. 4 Sensed mass by the open loop system $(O L)$, a closed loop system designed to detect humidity and temperature (CL1), and a closed loop system not designed to detect humidity and temperature $(C L 2)$ for a system with a change in humidity and with mass added at the tip (left), midspan (center) and both locations (right). Scenario 1 represents changes in mass at the tip. Scenario 2 represents changes in mass at the midspan. Scenario 3 represents changes in humidity. Scenario 4 represents changes in temperature.

and the $r$ index represents the number of parameters that can change $\mathbf{p}$, the $q$ index represents the number of measurable frequencies, and $\mathbf{S}$ is the sensitivity matrix. To determine the unknown changes in parameters from the known changes in frequencies, a pseudo-inverse of $\mathbf{S}$ in Eq. 6 can be used to yield

$$
\delta \mathbf{p}=S^{+} \delta \omega .
$$

The goal of sensitivity enhancement is to increase the entries in the closed loop sensitivity matrix $\mathbf{S}^{c i}$ with respect to the open loop sensitivity matrix $\mathbf{S}^{o}$. For a single closed loop system or for the open loop system, the solution of Eq. 7 is typically not very accurate because usually the number of changeable parameters $r$ is greater than the measurable frequencies $q$, which results in an under-determined problem. Koh and Ray ${ }^{21}$ overcame this problem by using multiple independent closed loop systems and unique combinations of actuator locations. Each closed loop system (of index $i$ ) corresponds to a unique sensitivity matrix $\mathbf{S}^{c i}$, which means that a complete closed loop sensitivity matrix can be expressed as

$$
\mathbf{S}^{c}=\left[\begin{array}{c}
\mathbf{S}^{c 1} \\
\mathbf{S}^{c 2} \\
\vdots \\
\mathbf{S}^{c t}
\end{array}\right]
$$

where $t$ corresponds to the number of unique controller configurations used. Therefore, if $t \cdot q>r$ then Eq. 7 becomes an over-determined set of equations for the unknowns $\delta$ p.

In this work, the parameter changes explored were both global and local. The local changes are variations of the mass of the beam in the vicinity of its tip and midspan (which correspond to sensing scenarios). The global changes are proportional variations in the entire mass or stiffness of the physical system. These scenarios correspond to changes in humidity (uniform changes in mass) and temperature (uniform changes in stiffness). The ability to distinguish the effects of humidity and temperature from the changes due to local additions of mass is critical for practical uses of this method for sensing and damage detection.

\section{Frequency Extraction for Augmented Systems}

In this section, a procedure is presented for the extraction of augmented resonant frequencies from a system with few measurement locations and noisy data. The procedure is specially designed for augmented linear systems. However, with a few modifications, it can be used for standard linear systems also.

The first step in the procedure is to excite the system at a single frequency within the frequency range of interest. This frequency range corresponds to the (placed) frequencies of the closed loop augmented system. The response of the system and the physical excitation is stored. Multiple measurements can be performed (at the same frequency), and the measured response and excitation can be averaged at each phase of the dynamics. Hence, noise can be largely filtered out. That is particularly useful when the response of the system is periodic. Next, the harmonic excitation is repeated for additional frequencies until enough frequency information has been extracted from the system.

The rest of the procedure deals with post processing and the actual extraction of the resonant frequencies. First, the augmented variables and the augmented forcing are constructed using the filtered data. The augmented variable $\mathbf{y}$ is computed directly from its nonlinear relation to $\mathbf{x}$, while $\ddot{\mathbf{y}}$ is calculated by finite differencing $\mathbf{y}$, and $\mathbf{h}$ is calculated directly from the left hand side of Eq. 3. Next, the full responses of the system ( $\mathbf{x}$ and $\mathbf{y}$ ) and full forcing ( $\mathbf{g}$ and $\mathbf{h}$ ) can be summed for all excitation frequencies to form a single (complex) excitation and response data set. The input ( $\mathbf{g}$ and $\mathbf{h}$ ) and output ( $\mathbf{x}$ and $\mathbf{y}$ ) can then be fed into DSPI, ${ }^{28}$ and the augmented frequencies can be extracted.

A specialized nonlinearity was designed for use in the nonlinear controller. It had the form $y=x^{3} \exp \left(-x^{2} / C\right)$ and a plot for $C=1$ is plotted in Fig. 2. The constant $C$ is a scaling term that can be used to adjust the maximum amplitude and width of the signal. There are several key features that make this nonlinearity desir- 

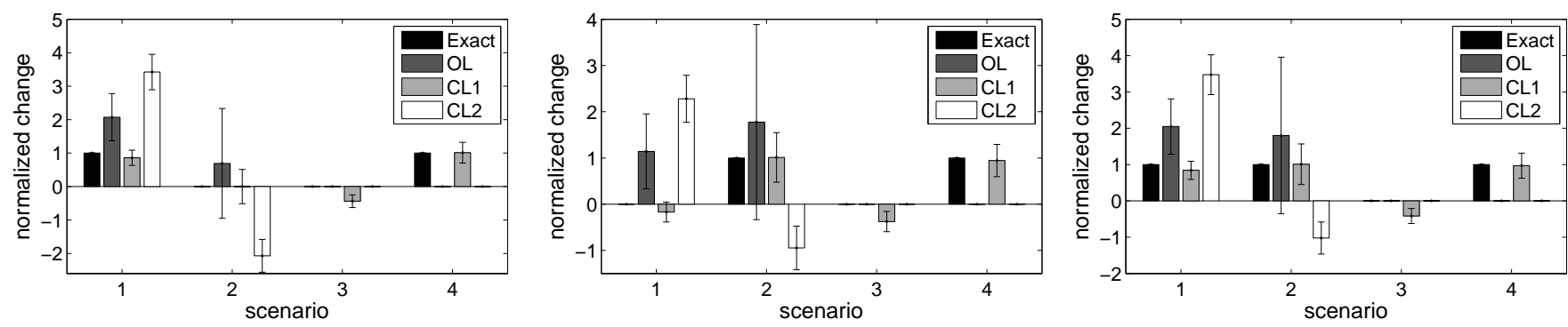

Fig. 5 Sensed mass by the open loop system $(O L)$, a closed loop system designed to detect humidity and temperature ( $C L 1)$, and a closed loop system not designed to detect humidity and temperature (CL2) for a system with a change in temperature and with mass added at the tip (left), midspan (center) and both locations (right). Scenario 1 represents changes in mass at the tip. Scenario 2 represents changes in mass at the midspan. Scenario 3 represents changes in humidity. Scenario 4 represents changes in temperature.

able. First, this is a smooth nonlinearity, and its second derivative can be calculated accurately using finite differencing. Second, the peak amplitude of the variable $y$ can be tailored to be close to the amplitude of the linear degrees of freedom (by choosing an adequate value for $C)$. Finally, the last key feature is that the nonlinear response tends to zero for large amplitudes in the system. This feature is important in making sure that the system response remains bounded during its interrogation. Since the nonlinearity exponentially tends to zero at large $x$, and the open loop (physical) linear system is stable, the closed loop system system returns toward the origin when the response becomes large.

\section{Numerical Results}

To demonstrate the proposed method, a numerical investigation was performed on a linear cantilevered beam shown in Fig. 1. The properties of the system are the same as the system investigated by Jiang et al. ${ }^{22}$ The density and Young's modulus of the beam are $2410 \mathrm{~kg} / \mathrm{m}^{3}$ and $6.6 \cdot 10^{10} \mathrm{~N} / \mathrm{m}^{2}$, respectively. The length, thickness, and width of the beam are $0.4 \mathrm{~m}$, $3.4 \mathrm{~mm}$, and $26 \mathrm{~mm}$. The density and Young's modulus of the piezoelectric material are $7600 \mathrm{~kg} / \mathrm{m}^{3}$ and $5.9 \cdot 10^{10} \mathrm{~N} / \mathrm{m}^{2}$, respectively. The length, thickness, and width of the piezoelectric patch are $40 \mathrm{~mm}, 0.3 \mathrm{~mm}$, and $20 \mathrm{~mm}$. The piezoelectric constant is $d_{31}=-276$. $10^{-12} \mathrm{~m} / \mathrm{V}$. The beam was discretized into 10 elements with the control and forcing input to the system applied through a moment induced by the piezoelectric patch on the second element of the beam. A light proportional damping of the form $\alpha \mathbf{M}+\beta \mathbf{K}$ was also added to the beam, where $\alpha=10^{2}$ and $\beta=10^{-5}$. Five position measurements (out of a possible 20 degrees of freedom for the system model) were taken along the beam, as indicated in Fig. 1.

In addition to the 5 physical measurements, 5 augmented variables were created for the system. The augmented variables correspond to $y_{i}=x_{i}^{3} \exp \left(-x_{i}^{2} / C\right)$, where $x_{i}$ are the 5 measured signals. Only these nonlinearities are used in the controller. The augmented system was created by generating $\mathbf{M}, \mathbf{K}, \mathbf{N}_{I}, \mathbf{N}_{S}, \mathbf{N}_{A I}$,
$\mathbf{N}_{A D}, \mathbf{N}_{A S}$ and $\mathbf{N}_{C S}$ as discussed in the system augmentation section. The augmented matrices and control gains were optimized using the "fmincon" function in MATLAB ${ }^{29}$ following the optimization algorithm laid out in. ${ }^{24}$

In general, the user has complete control over the 5 augmented degrees of freedom corresponding to the 5 augmented variables. Hence, multiple independent closed loop configurations are possible (even though there is only one physical controller). In this work, the only controller configuration that was used corresponds to control at the physical degrees of freedom affected by the piezoelectric patch and the 5 augmented degrees of freedom.

The first 5 resonant frequencies of the system were optimally placed to maximize the sensitivity and independence of 4 scenarios. The first scenario corresponds to added mass at the tip of the beam. The second scenario corresponds to added mass at the midspan of the beam. The third scenario corresponds to a change in humidity of the environment (characterized by a uniform change in mass of the physical system). The fourth (and last) scenario corresponds to a change in temperature of the environment (characterized by a uniform change in stiffness of the physical system).

Two closed loop controllers were designed. The first one, denoted by $C L 1$, was designed to detect variations in humidity and temperature as well as added mass at the tip and midspan of the beam. The second controller, denoted by $C L 2$, was designed to detect only added mass at the tip and midspan of the beam.

To demonstrate the frequency extraction method, the eigenvalues of the healthy closed loop system $(C L 1)$ were extracted by DSPI for the case of zero noise and for the case of noise with a Gaussian distribution having zero mean and a standard deviation of about $0.5 \%$ of the the response (and excitation) of the system. The results are shown in Table 1. The first column consists of the first seven exact eigenvalues. The second column consists of the eigenvalues extracted by DSPI for the noise free case. The third column contains the average values of the eigenvalues obtained from 100 separate numerical 

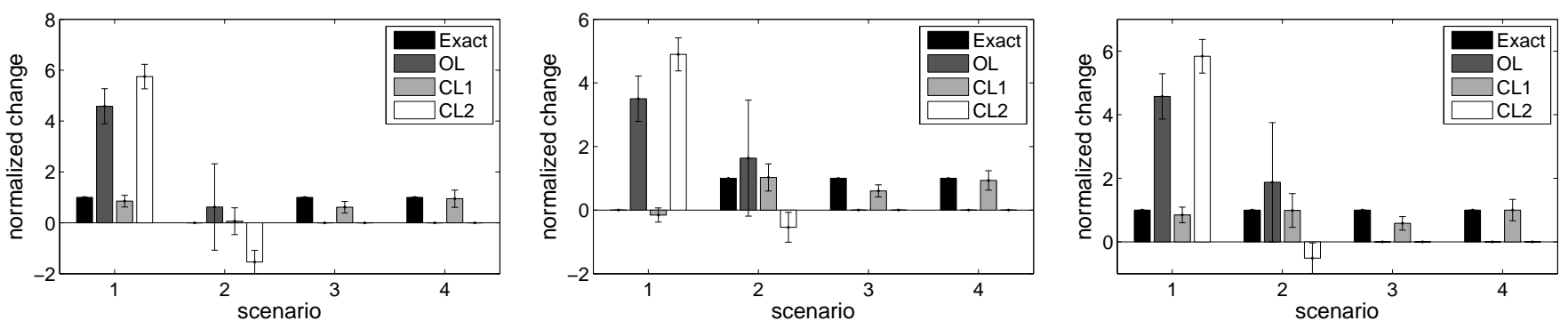

Fig. 6 Sensed mass by the open loop system $(O L)$, a closed loop system designed to detect humidity and temperature $(C L 1)$, and a closed loop system not designed to detect humidity and temperature $(C L 2)$ for a system with a change in humidity and temperature, and with mass added at the tip (left), midspan (center) and both locations (right). Scenario 1 represents changes in mass at the tip. Scenario 2 represents changes in mass at the midspan. Scenario 3 represents changes in humidity. Scenario 4 represents changes in temperature.

simulations for the noisy case. The fourth and last column consists of the standard deviations obtained for the identified frequencies. Comparing the first two columns one may note that DSPI can very accurately extract the augmented eigenvalues of the system for zero noise. In the presence of noise, the method still works quite well with the average value very close to the exact eigenvalue with small deviations.

For the following results, the exact frequencies were calculated for the system, and a noise with a Gaussian distribution having zero mean was added to the eigenvalues of the system. The standard deviation for the noise distribution was approximately equal to the standard deviations for the frequencies given in Table 1 . The first eigenvalue was not used in the analysis since the noise level in that eigenvalue was significantly greater than the other 4 placed eigenvalues.

The results in Fig. 3 show the changes predicted for 3 different systems in 3 cases, when there is no change in the humidity or temperature of the system from their nominal value. The plot on the left corresponds to a case where a mass of $0.1 \%$ of the beam is at the tip of the beam. The plot in the center corresponds to the case where a mass of $0.1 \%$ of the beam is at the midspan of the beam. The plot on the right corresponds to the case where there are masses each of $0.1 \%$ of the beam at both the tip and the midspan of the beam. There are 4 bars plotted for each scenario. The first bar is the exact change in the system. The rest of the bars have standard deviation error bars for the noisy cases. The second bar is the value predicted by an open loop system. The sensitivity matrix of the open loop system would be rank deficient if it was taking into account temperature and humidity effects. Therefore, a sensitivity matrix based solely on added mass at the tip and midspan was created (in the same way as for $C L 2$ ). The third bar is the change predicted by $C L 1$. The fourth and final bar is the change predicted by $C L 2$. The open loop predictions of the mass variations are quite poor. In this case, $C L 2$ slightly outperforms $C L 1$ since there was no change in the temperature or humidity.

The results in Fig. 4 show the changes predicted for the same 3 systems and the same cases as in Fig. 3. However, there is a change in humidity that causes a $1 \%$ change in the mass of the system. For this scenario, the mass variations are once again poorly detected by the open loop system. Additionally, $C L 2$ performs poorly in comparison to $C L 1$ since it is not designed to handle changes in the humidity of the environment.

The results in Fig. 5 are similar to the results in Fig. 4. However, instead of a change in humidity, there is a change in temperature that causes a $1 \%$ change in the stiffness of the system. For this scenario, the mass variations are once again poorly detected by the open loop system. Additionally, $C L 2$ performs poorly in comparison to $C L 1$ since it is not designed to handle changes in the temperature of the environment.

The results in Fig. 6 are similar to the results in Fig. 5 and Fig. 4. However, in this scenario there is a $1 \%$ change in both the mass and stiffness of the system due to changes in temperature and humidity. Once again the open loop system performs poorly as well as $C L 2$, which was not designed to handle humidity or temperature changes. However, $C L 1$ is able to accurately sense the added mass for the 3 different scenarios even in the presence of noise.

\section{Conclusions}

A novel approach for sensitivity enhancement for linear and nonlinear systems via optimal augmentations and nonlinear feedback auxiliary signals was presented. This approach extends previous work by detailing a methodology for augmented frequency extraction using noisy and limited measurement data. Several advantages of nonlinear feedback auxiliary signals over traditional linear feedback were detailed. In particular, the ability to control all the augmented degrees of freedom without actuation effort (just signal processing) were exploited. Also, the additional information corresponding to the augmented variables was shown to play an important role when there is limited measurement data. In particular, the additional measurement information enables the placement of additional resonant frequencies. Another advantage of the augmented system is that it does 
not have to be stable (although the physical linearized system must be stable).

Sensitivity enhancement has been demonstrated in a linear cantilevered beam controlled with a single piezoelectric patch and measuring only 5 degrees of freedom using feedback auxiliary signals and system augmentation. The method is able to isolate temperature and humidity effects from multiple simultaneous mass variations. The masses detected were $0.1 \%$ of the mass of the physical beam. Numerical simulations were conducted with limited measurements and noisy data.

\section{Acknowledgments}

The authors wish to acknowledge the National Science Foundation (CAREER and Graduate Research Fellowship programs) for the generous support of this work.

\section{References}

${ }^{1}$ Wolf, K. and Gottlieb, O., "Nonlinear Dynamics of a Noncontacting Atomic Force Microscope Cantilever Actuated by a Piezoelectric Layer," Journal of Applied Physics, Vol. 91, No. 7, 2002, pp. 4701-4709.

${ }^{2}$ DeVoe, D., "Piezoelectric Thin Film Micromechanical Beam Resonators," Sensors and Actuators A, Vol. 88, No. 2, 2001, pp. 263-272.

${ }^{3}$ Ilic, B., Czaplewski, D., Zalalutdinov, M., Craighead, H. G. Neuzil, P., Campagnolo, C., and Batt, C., "Single Cell Detection with Micromechanical Oscillators," Journal of Vacuum Science and Technology, Vol. 19, No. 6, 2001, pp. 2825-2828.

${ }^{4}$ Thundat, T., Wachter, E. A., Sharp, S. L., and Warmack, R. L., "Detection of Mercury-Vapor Using Resonating Microcantilevers," Applied Physics Letters, Vol. 66, No. 13, 1995.

${ }^{5}$ Chen, G. Y., Thundat, T., Wachter, E. A., and Warmack, R. J., "Adsorption-Induced Surface Stress and its Effects on Resonance Frequency of Microcantilevers," Journal of Applied Physics, Vol. 77, No. 8, 1995

${ }^{6}$ Doebling, S. W., Farrar, C. R., Prime, M. B., and Shevitz, D. W., Damage identification and health monitoring of strucutural and mechanical systems from changes in their vibration characteristics: a literature review, Los Alamos National Laboratory Report LA-13070-MS, Los Alamos, NM, 1996.

${ }^{7}$ Doebling, S. W., Farrar, C. R., and Prime, M. B., "A summary review of vibration-based damage identification methods," Shock and Vibration Digest, Vol. 30, No. 2, 1998, pp. 91-105.

${ }^{8}$ Cornwell, P., Kan, M., Carlson, B., Hoerst, L. B., Doebling, S. W., and Farrar, C. R., "Comparative study of vibration-based damage identfication algorithms," Proceedings of the 16th International Modal Analysis Conference, Part 2, Santa Barbara, CA, 1998, pp. 1710-1716.

${ }^{9}$ Friswell, M. I. and Penny, J., "The practical limits of damage detection and location using vibration data," Proceedings of the 11th VPI \&S SU Symposium on Structural Dynamics and Control, Blacksburg, VA, 1997, pp. 31-40.

${ }^{10}$ Salawu, O. S., "Detection of structural damage through changes in frequency : a review," Engineering Structures, Vol. 19, No. 9, 1997, pp. 718-723.

${ }^{11}$ Stubbs, N. and Osegueda, R., "Global non-destructive damage evaluation in solids," Modal Analysis: The International Journal of Analytical and Experimental Modal Analysis, Vol. 5, No. 2, 1990, pp. 67-79.

${ }^{12}$ Stubbs, N. and Osegueda, R., "Global damage detection in solids-experimental verification," Modal Analysis: The International Journal of Analytical and Experimental Modal Analysis, Vol. 5, No. 2, 1990, pp. 81-97.

${ }^{13}$ Nalitolela, N. G., Penny, J. E. T., and Friswell, M. I., "Mass or stiffness addition technique for strucutral parameter updating,"
Modal Analysis: The International Journal of Analytical and Experimental Modal Analysis, Vol. 7, No. 3, 1992, pp. 157-168.

${ }^{14}$ Lew, J. S. and Juang, J. N., "Structural damage detection using virtual passive controllers," Journal of Guidance, Control, and Dynamics, Vol. 25, No. 3, 2002, pp. 419-424.

${ }^{15}$ Swamidas, A. S. J. and Chen, Y., "Monitoring crack growth through change of modal parameters," Modal Analysis: The International Journal of Analytical and Experimental Modal Analysis, Vol. 5, No. 2, 1990, pp. 67-79.

${ }^{16}$ Adams, R. D., Cawley, P., Pye, C. J., and Stone, B. J., "A vibrational technique for non-destructively assessing the integrity of structures," Journal of Mechanical Engineering Science, Vol. 20 , No. 2, 1978, pp. 93-100.

${ }^{17}$ Ray, L. R. and Tian, L., "Damage detection in smart structures using sensitivity enhancing feedback control," Journal of Sound and Vibration, Vol. 227, No. 5, 1999, pp. 987-1002.

${ }^{18}$ Ray, L. R., Koh, B. H., and Tian, L., "Damage detection and vibration control in smart plates: Towards multifunctional smart structures," Journal of Intelligent Material Systems and Structures, Vol. 11, No. 9, 2000, pp. 725-739.

${ }^{19}$ Ray, L. R. and Marini, S., "Optimization of control laws for damage detection in smart structures," SPIE Symposium on Mathematics and Control in Smart Structures, Vol. 3984, 2000, pp. 395-402.

${ }^{20}$ Juang, J. N., Lim, K. B., and Junkins, J. L., "Robust Eigensystem Assignment for Flexible Structures," Journal of Guidance, Vol. 12, No. 3, 1989, pp. 381-387.

${ }^{21}$ Koh, B. H. and Ray, L. R., "Feedback controller design for sensitivity-based damage localization," Journal of Sound and Vibration, Vol. 273, No. 1-2, 2004, pp. 317-335.

22 Jiang, L. J., Tang, J., and Wang, K. W., "An optimal sensitivity-enhancing feedback control approach via eigenstructure assignment for structural damage identification," ASME Journal of Vibration and Acoustics, Vol. 129, No. 6, 2007, pp. 771-783.

${ }^{23}$ D'Souza, K. and Epureanu, B. I., "Damage Detection in Nonlinear Systems Using Optimal Feedback Auxiliary Signals," ASME Journal of Vibration and Acoustics, submitted, 2007.

${ }^{24}$ D'Souza, K. and Epureanu, B. I., "Nonlinear Feedback Auxiliary Signals for System Interrogation and Damage Detection," Philosophical Transactions of the Royal Society of London: A - Mathematical, Physical and Engineering Sciences, submitted, 2008.

${ }^{25}$ D'Souza, K. and Epureanu, B. I., "Damage detection in nonlinear systems using system augmentation and generalized minimum rank perturbation theory," Smart Materials and Structures, Vol. 14, No. 5, 2005, pp. 989-1000.

${ }^{26}$ D'Souza, K. and Epureanu, B. I., "Multiple augmentations of nonlinear systems and generalized minimum rank perturbations for damage detection," Journal of Sound and Vibrations, accepted, 2006.

${ }^{27}$ D'Souza, K. and Epureanu, B. I., "Sensor placement for damage detection in nonlinear systems using system augmentations," AIAA Journal, submitted, 2007.

${ }^{28}$ Leuridan, J., Some Direct Parameter Model Identification Methods Applicable For Multiple Modal Analysis, PhD thesis, Department of Mechanical and Industrial Engineering, University of Cincinnati, 1984.

${ }^{29}$ Coleman, T., Branch, M. A., and Grace, A., Opimization Toolbox User's Guide, The MathWorks, Inc., $3^{\text {rd }}$ ed., 1999. 